\title{
Conhecimento e Abordagens acerca do Uso Problemático do Álcool
}

\author{
Fernando Gomes Reis \\ Universidade de Franca, SP, Brasil. \\ Mônica de Andrade \\ Universidade de Franca, SP, Brasil.
}

\author{
Everson Cardozo Machado \\ Universidade Federal Fluminense, RJ, Brasil. \\ Glória Lúcia Alves Figueiredo \\ Universidade de Franca, SP, Brasil.
}

Resumo: A ocorrência de mortes e adoecimentos relacionados ao consumo de bebidas alcoólicas é relevante em todo mundo e provoca custos altíssimos para a população. Trata-se de um estudo de intervenção, qualitativo e quantitativo, com objetivo de avaliar a efetividade de um curso de atualização para os profissionais que atuam na Atenção Primária à Saúde (APS) acerca do conhecimento e das formas de abordagens frente ao uso problemático do álcool em um município mineiro. O conteúdo do curso teve os constructos da Promoção de Saúde como referencial teórico. Aplicou-se um questionário com perguntas fechadas relativas ao conteúdo trabalhado, antes e após o curso. A amostra foi composta por 39 participantes trabalhadores da APS. Os resultados revelaram que, após o curso, o percentual total de acertos foi de 81,0\%, evidenciando uma evolução de $11,4 \%$ do número de respostas corretas. Conclui-se que o modelo de intervenção composto por curso que aborda temas do cotidiano mais encontros com debates e espaço para a participação dos profissionais é uma proposta viável para atualização dos profissionais que atuam frente à problemática do consumo de bebidas alcoólicas. A proposta apresenta, ainda, uma possibilidade de atuação do profissional da Psicologia na rede municipal de saúde com foco nos constructos da Promoção de Saúde.

Palavras-chave: Atenção Primária à Saúde, Saúde Mental, Transtornos Relacionados ao Uso de Álcool, Promoção da Saúde.

\section{Knowledge and Approaches on the Issue of Alcohol Consumption}

Abstract: The occurrence of deaths and illnesses related to alcohol consumption is relevant throughout the world, causing exorbitant costs for the population. This is a qualitative and quantitative intervention study in order to analyze the effectiveness of a refresher course for professionals working with Primary Health Care (PHC) as regards knowledge and means of approach on the issue of alcohol consumption in a city of the State of Minas Gerais. The course followed the guidance of the constructs of Health Promotion as a theoretical framework. It was applied a questionnaire with closed questions regarding the content worked before and after the course. The sample consisted of 39 participants working with PHC. The results revealed that after the completion of the course the total percentage of correct answers was $81 \%$, showing an increase of $11.4 \%$ in the number of correct answers. We conclude that the intervention model that consists of a course addressing everyday topics, plus meetings with debates and space for the participation of professionals, is a viable proposal to refresh professionals facing the issue of alcohol consumption. The proposal also presents a possibility for the Psychology professional to work in the municipal health network with a focus on the Health Promotion constructs.

Keywords: Primary Health Care, Mental Health, Alcohol Use Disorders, Health Promotion. 


\title{
El Conocimiento y las Propuestas Presentadas acerca del Consumo Problemático de Alcohol
}

\begin{abstract}
Resumen: La ocurrencia de muertes y enfermedades relacionadas con el consumo de bebidas alcohólicas es relevante en todo el mundo, causando altos costos a la población. Se trata de un estudio de intervención, cualitativo y cuantitativo, con el fin de evaluar la efectividad de un curso de actualización para los profesionales que actúan en la Atención Primaria a la Salud (APS) acerca del conocimiento y de las propuestas presentadas ante el consumo problemático del alcohol en un municipio de Minas Gerais. El contenido del curso ha seguido la orientación de las construcciones de Promoción de la Salud como un marco teórico. Se aplicó un cuestionario con preguntas cerradas relativas al contenido trabajado antes y después del curso. La muestra se compuso de 39 participantes trabajadores de la APS. Los resultados revelaron que una vez concluido el curso, el porcentaje total de respuestas correctas fue de $81,0 \%$, con una evolución de $11,4 \%$ en el número de respuestas correctas. Llegamos a la conclusión de que el modelo de intervención que compone el curso plantea temas del cotidiano, además de los encuentros con los debates y el espacio para la participación de los profesionales, y es una propuesta viable para la actualización de los profesionales que actúan ante la problemática del consumo de bebidas alcohólicas. La propuesta también presenta la posibilidad de que el profesional de Psicología trabaje en la red municipal de salud enfocándose en los constructos de Promoción de la Salud.
\end{abstract}

Palabras clave: Atención Primaria de Salud, Salud mental, Trastornos Relacionados con el Consumo del Alcohol, Promoción de la Salud.

\section{Introdução}

O mundo lida com as consequências do uso do álcool há pelo menos oito mil anos. Considerada uma droga psicotrópica ou psicoativa por ser capaz de atravessar a barreira hematoencefálica e agir no sistema nervoso, o álcool causa alterações na consciência e é capaz de gerar mecanismos para a dependência de substâncias psicoativas (SENAD, 2014; Silva, 2005; WHO, 2010a).

Internacionalmente o álcool tem sido considerado um dos principais fatores de risco para a saúde. O seu uso prejudicial vem contribuindo para a carga global de doenças e, com relação a mortes prematuras e incapacidades, está listado como terceiro principal fator. O Global Strategy to Reduce the Harmful Use of Alcohol (WHO) ainda apontou a estimativa de que, em 2004, 2,5 milhões de mortes tiveram causas relacionadas com o álcool, incluindo 320 mil ocorridas entre jovens de 15 a 29 anos de idade (BAU, 2002; WHO, 2010a).

De acordo com a Agência Nacional de Saúde Suplementar, o álcool é uma das poucas drogas psicotrópicas que tem seu consumo admitido e incentivado pela sociedade. Nas últimas décadas, o seu consumo vem aumentando no mundo todo, sendo que a maior parte deste aumento se deve aos países em desenvolvimento. Calcula-se que, mundialmente, o álcool esteja relacionado a 3,2\% de todas as mortes. Os prejuízos causados pelo uso do álcool atingem a população desde a questão do trânsito, passando pela violência doméstica, absenteísmo no trabalho e onerosos gastos com a saúde pública em doenças decorrentes (ANS, 2011).

Sobre a população brasileira, o II Levantamento Nacional de Álcool e Drogas (Lenad) apontou que as taxas de abstinência permaneceram com diferença não significativa entre 2006 e 2012, mantendo-se em torno de $50 \%$. Houve aumento na proporção de bebedores frequentes (20\%), que são os que bebem uma vez por semana ou mais. E o levantamento também chamou atenção para o aumento do modo de beber nocivo, ou seja, a ingestão de quatro unidades de álcool para mulheres e cinco unidades para homens em um período igual ou inferior a duas horas (Laranjeira, Madruga, \& Pinsku, 2014).

No Brasil, dentre os níveis de complexidade, a atenção primária ou básica à saúde (APS) é considerada a porta de entrada para os serviços de saúde no 
Sistema Único de Saúde (SUS). Tem como finalidade a descentralização das ações de saúde, a garantia do acesso e atenção aos outros níveis de complexidade a toda população (Brasil, 2006).

A legislação brasileira preconiza estratégias de estímulo à inserção de ações de promoção da saúde com ênfase na APS, desenvolvimento de estratégias de qualificação para profissionais de saúde inseridos no SUS e apoio a projetos consonantes com a realidade local para profissionais que atuem na Estratégia Saúde da Família (ESF) (Brasil, 2006).

Ainda compondo o Departamento de Atenção Básica (DAB), os Núcleos de Apoio à Saúde da Família (NASF) foram criados em 2008 com o objetivo de intensificar a resolubilidade das ações da atenção básica à saúde, qualificando as intervenções nos territórios onde reside a população adscrita e, entre outras atribuições, possibilitando parcerias intersetoriais para a evolução dos serviços (Olschowsky, Wetzel, Fernando, Pinho, \& Camatta, 2014.

As redes de saúde municipais os Centros de Atenção Psicossocial (CAPS) devem se responsabilizar pela organização da demanda e da rede de cuidados em saúde mental no âmbito do seu território, além de supervisionar e capacitar as equipes da APS, bem como serviços e programas de saúde mental (Brasil, 2002).

Cabe ressaltar que a dependência de substâncias psicoativas raramente é incluída como parte da proposta curricular em cursos de graduação. Observa-se a existência de uma conotação moral na avaliação dos casos que envolvem o uso abusivo e prejudicial de substâncias psicoativas, fazendo-se necessária reflexão sobre a prática e o enfrentamento destas questões cotidianas do trabalho na APS (Lima, 2014; Silva, 2005; Souza, 2014).

Para tal, esse estudo se justifica, pois, o conhecimento sobre o consumo de bebidas alcoólicas e a abordagem das pessoas acometidas por estas demandas são fundamentais para a eficiência do trabalho e a coerência com os princípios que orientam o SUS. Ainda divulga uma possibilidade de atuação do profissional da Psicologia inserido na Rede de Atenção Psicossocial (RAPS) do município, neste caso, em um CAPS.

Partindo do pressuposto de que para a evolução dos serviços de saúde se faz necessário o aprimoramento contínuo dos profissionais com a promoção de encontros e discussões no formato de cursos, este estudo tem como objetivo avaliar a efetividade de um curso de atualização para os profissionais que atuam na APS acerca do conhecimento (consumo, políticas e serviços de saúde) e das formas de abordagens frente ao uso problemático do álcool em um município mineiro.

\section{Método}

Trata-se de um estudo experimental, do tipo prée pós-intervenção de abordagem quantitativa e qualitativa com um grupo de profissionais que participaram de um curso e foram avaliados e comparados em dois momentos. O pré-teste tem por objetivo investigar as noções iniciais ou espontâneas que os participantes apresentam. O pós-teste objetiva examinar as noções apresentadas pelos participantes após a intervenção experimental (Spinillo, 1994). O estudo foi aprovado pelo Comitê de Ética em Pesquisa da Universidade de Franca sob o no 39132214.0.0000.5495. Foram convidados 50 profissionais das cinco equipes da ESF e de uma equipe do NASF do município de estudo. Desses, oito não aceitaram participar e três não compareceram no primeiro dia do curso, compondo uma amostra de 39 participantes que assinaram o Termo de Consentimento Livre e Esclarecido (TCLE). Aos participantes foi atribuída a letra $P$ seguida de um número para garantia de sigilo.

Foi elaborado um curso de seis horas, divido em três módulos, programados para três encontros semanais de duas horas cada. Os temas dos encontros abordaram o uso, o abuso e a dependência de álcool e foram escolhidos de forma intencional. Acredita-se que, revisitando a fundamentação teórica que embasa a prática dos profissionais de saúde participantes do estudo, pode-se provocar intervenções mais adequadas. O conteúdo teórico seguiu os constructos da Promoção de Saúde, literatura científica, documentos governamentais, sites e ainda foram acrescentados imagens e links de vídeos que foram apresentados no decorrer dos encontros. Ao início do curso cada participante recebeu o material didático impresso.

Os encontros aconteceram na Câmara Municipal, devidamente reservada para esse fim. O primeiro módulo iniciou com a temática: $\mathrm{O}$ uso de bebidas alcoólicas, abordando definição, histórico e epidemiologia do uso do álcool; políticas públicas; promoção de saúde; e APS. O tema do segundo módulo foi: Efeitos do álcool e sistemas classificatórios, abordando padrões de uso; transtornos mentais e comportamentais devido ao uso do álcool; e modalidades de tratamento e estágios motivacionais. O terceiro e 
último módulo enfocou o tema Abordagens e conhecimentos, tratando dos seguintes assuntos: abordagens e dificuldades; rastreamento e identificação.

Todas as etapas foram conduzidas e ministradas por um dos pesquisadores que exercia a função de psicólogo no CAPS do município. As exposições teóricas foram participativas com discussão e apresentação de estudos de casos. Em cada módulo foi programado um tempo para o diálogo e debate dos temas. A programação foi influenciada pela proposta de Educação Continuada em Saúde de modo a valorizar e debater conteúdos vivenciados pelos cursistas no cotidiano do trabalho e relacioná-los com diferentes conhecimentos acadêmicos divulgados sobre $o$ assunto.

A Organização Panamericana da Saúde (OPS) define educação continuada como um processo dinâmico de ensino aprendizagem, ativo e permanente, destinado a atualizar e melhorar a capacidade de pessoas, ou grupos, face às necessidades sociais, aos objetivos e metas das instituições. A educação continuada inclui as experiências educacionais que possam aumentar a base de conhecimentos e habilidades. Parte da reflexão sobre a realidade do serviço e das necessidades existentes para então formular estratégias que ajudem a solucionar problemas, articulando responsabilidade, liberdade e compromisso (Oguisso, 2000; OPS, 1979; Silva, \& Seiffert, 2009).

Portanto, a metodologia aplicada buscou distanciar-se de uma proposta mecânica e pouco interativa, na tentativa de promover o desafio sobre o que é possível mudar na realidade de trabalho com os conhecimentos teóricos produzidos até o momento (Pereira, \& Lima, 2006).

Para coleta de dados, foi utilizado um diário de campo para anotações gerais e dois questionários autoaplicáveis, um com os dados sociodemográficos e outro com perguntas do tipo múltipla escolha, abordando o conteúdo programático do curso. Este último foi aplicado em dois momentos, antes e imediatamente após o término do curso proposto. Os questionários foram tabulados separadamente nos dois momentos.

O questionário utilizado foi composto por 20 questões de múltipla escolha. Algumas questões foram elaboradas pelo pesquisador e outras foram adaptadas ou replicadas na íntegra do Programa Supera (Sistema Para Detecção do Uso Abusivo e Dependência de Substâncias Psicoativas: Encaminhamento, Intervenção Breve, Reinserção Social e Acom- panhamento) promovido pela Secretaria Nacional de Políticas sobre Drogas (Senad) do Ministério da Justiça (MJ). Para efeito de análise as questões foram agrupadas e classificadas em: Consumo de álcool; Políticas e serviços de saúde; e Abordagem profissional.

A utilização do diário de campo teve o intuito de articular o aprofundamento teórico com o processo em análise para o embasamento da discussão. Este instrumento auxiliou na sistematização, detalhamento e registros das falas e situações ocorridas durante o curso. As observações sobre os relatos foram registradas. E o diário foi estruturado em descrição e interpretação do observado, bem como com os registros das conclusões, dúvidas e desafios apresentados, de modo a possibilitar relações entre os dados qualitativos e quantitativos (Falkembach, 1987; Lima, Mioto e Dal Prá, 2007).

Considerando as características do estudo, a análise dos dados quantitativos foi feita utilizando-se o pacote estatístico Statical Package for Social Sciences (SPSS 22). As variáveis categóricas foram expressas por valor absoluto ou percentual e as contínuas, por média \pm desvio-padrão.

\section{Resultados}

A amostra foi composta por 39 participantes, que apresentaram na Tabela 1 o seu perfil sociodemográfico.

A média de idade dos participantes foi de 33,2 anos, sendo a maioria do sexo feminino $(92,3 \%)$, casados ou em relação estável (53,9\%), com ensino médio completo (59\%). Entre as categorias profissionais os agentes comunitários de saúde, cirurgiões

\section{Tabela 1}

Perfil sociodemográfico da amostra de estudo, 2015.

\begin{tabular}{lcc}
\hline Variáveis & $\mathrm{n}(39)$ & $\%$ \\
\hline Sexo & 36 & 92,3 \\
Feminino & 3 & 7,7 \\
Masculino & 14 & 35,9 \\
Solteiro & 4 & 10,2 \\
Divorciado/viúvo & & \\
Escolaridade & 2 & 5,1 \\
Ensino fundamental completo & 23 & 59 \\
Ensino médio completo & 11 & 28,2 \\
Ensino superior completo & 3 & 7,7 \\
Pós-graduação & & \\
\hline
\end{tabular}


dentistas, enfermeiros, farmacêuticos, fisioterapeutas, médicos, nutricionistas, psicólogos, técnicos em enfermagem, técnicos em saúde bucal e terapeutas ocupacionais compuseram a amostra.

Os resultados do questionário aplicado em dois momentos, antes e após o curso de atualização, estão demonstrados na Tabela 2.
Com relação às questões acerca do Consumo de Álcool, dentre as seis questões propostas, cinco apresentaram maior frequência de acertos no segundo momento de aplicação. Destaca-se principalmente a pergunta $\mathrm{n}^{0} 4$ com a definição do conceito de dose-padrão que apresentou aumento da frequência de respostas corretas de $42,5 \%$.

Tabela 2

Percentual de desempenho dos profissionais antes e depois do curso de atualização referente ao conhecimento sobre o consumo de álcool, políticas e serviços de saúde e abordagem profissional, 2015.

\begin{tabular}{|c|c|c|c|c|c|}
\hline \multirow[b]{2}{*}{ Categoria } & \multicolumn{2}{|c|}{ Antes $(\mathrm{n}=39)$} & \multicolumn{2}{|c|}{ Depois $(\mathrm{n}=37)$} & \multirow{2}{*}{$\begin{array}{c}\text { Diferença } \\
(\%)\end{array}$} \\
\hline & $\begin{array}{c}\text { Frequência } \\
\text { acertos }\end{array}$ & $\begin{array}{c}\text { Percentual } \\
(\%)\end{array}$ & $\begin{array}{l}\text { Frequência } \\
\text { acertos }\end{array}$ & $\begin{array}{c}\text { Percentual } \\
(\%)\end{array}$ & \\
\hline \multicolumn{6}{|l|}{ Consumo de álcool } \\
\hline Questão 1 & 28 & 73,7 & 31 & 86,1 & 12,4 \\
\hline Questão 2 & 29 & 74,4 & 33 & 91,7 & 17,3 \\
\hline Questão 3 & 14 & 38,9 & 18 & 50 & 11,1 \\
\hline Questão 4 & 17 & 43,6 & 31 & 86,1 & 42,5 \\
\hline Questão 5 & 36 & 92,3 & 32 & 88,9 & $-3,4$ \\
\hline Questão 15 & 5 & 13,9 & 14 & 38,9 & 25 \\
\hline Subtotal de acertos & \multicolumn{2}{|c|}{129} & \multicolumn{2}{|c|}{159} & \\
\hline Percentual (\%) & \multicolumn{2}{|c|}{56,8} & \multicolumn{2}{|c|}{73,6} & 16,8 \\
\hline \multicolumn{6}{|c|}{ Políticas e serviços de saúde } \\
\hline Questão 6 & 32 & 82,1 & 33 & 94,3 & 12,2 \\
\hline Questão 7 & 15 & 38,5 & 27 & 75 & 36,5 \\
\hline Questão 8 & 38 & 97,4 & 36 & 100 & 2,6 \\
\hline Questão 11 & 36 & 92,3 & 34 & 94,4 & 2,1 \\
\hline Questão 13 & 16 & 42,1 & 26 & 72,2 & 30,1 \\
\hline Questão 16 & 36 & 94,7 & 34 & 94,4 & $-0,3$ \\
\hline Questão 18 & 28 & 73,7 & 29 & 80,6 & 6,9 \\
\hline Subtotal de acertos & \multicolumn{2}{|c|}{201} & \multicolumn{2}{|c|}{219} & \\
\hline Percentual (\%) & \multicolumn{2}{|c|}{74,4} & \multicolumn{2}{|c|}{87,3} & 12,8 \\
\hline \multicolumn{6}{|l|}{ Abordagem profissional } \\
\hline Questão 9 & 34 & 87,2 & 32 & 88,9 & 1,7 \\
\hline Questão 10 & 36 & 94,7 & 36 & 100 & 5,3 \\
\hline Questão 12 & 37 & 94,9 & 36 & 100 & 5,1 \\
\hline Questão 14 & 32 & 84,2 & 31 & 91,2 & 7 \\
\hline Questão 17 & 25 & 65,8 & 29 & 80,6 & 14,8 \\
\hline Questão 19 & 16 & 41 & 8 & 22,2 & $-18,8$ \\
\hline Questão 20 & 24 & 61,5 & 31 & 86,1 & 24,6 \\
\hline Subtotal de acertos & \multicolumn{2}{|c|}{204} & \multicolumn{2}{|c|}{203} & \\
\hline Percentual (\%) & \multicolumn{2}{|c|}{75,6} & \multicolumn{2}{|c|}{81,2} & 5,6 \\
\hline Total de Acertos & \multicolumn{2}{|c|}{534} & \multicolumn{2}{|c|}{581} & \\
\hline Percentual (\%) & \multicolumn{2}{|c|}{69,6} & \multicolumn{2}{|c|}{81} & 11,4 \\
\hline
\end{tabular}


Em relação às questões sobre Políticas e Serviços de Saúde, dentre sete propostas, seis apresentaram maior frequência de acertos. Com a análise estatística, sobressai a questão $\mathrm{n}^{\circ} 8$, que abordou os objetivos da Estratégia Saúde da Família e obteve um aumento do número de acertos de $36,5 \%$. Os resultados revelam que após a realização do curso os participantes acertaram 87,3\% das questões acerca das Políticas e Serviços de Saúde.

Sete questões também compuseram a temática da Abordagem Profissional, sendo que nesta categoria as diferenças entre os acertos antes e depois foram menos significativas, entretanto, justamente nesta categoria, $75,6 \%$ de acertos foram contabilizados já no primeiro momento da aplicação.

Evidencia-se uma evolução de $11,4 \%$ do número de respostas corretas. Enquanto o percentual total de acertos na aplicação anterior ao curso foi de 69,6\%, no segundo momento, após a realização do curso, o percentual total de acertos foi de $81,0 \%$. Os dados também revelaram elevado conhecimento prévio sobre assuntos abordados pelo curso, visto que o total de acertos na primeira aplicação se aproxima dos $70,0 \%$.

De modo geral, o conhecimento dos participantes sobre o uso problemático do álcool relacionado principalmente à atuação dos profissionais da Atenção Primária foi satisfatório visto que, dentre as 20 perguntas propostas, 12 apresentam frequências de acertos superiores a 70,0\% no primeiro dia de aplicação.

\section{Discussão}

Inicialmente, é importante destacar que todo o conteúdo do questionário foi abordado com os profissionais por meio de aula expositiva e distribuição de material educativo, além do incentivo ao diálogo a cada encontro acerca dos conteúdos programados.

O primeiro tema programado referiu-se ao dado de que aproximadamente $50 \%$ da população brasileira fez uso de álcool no último ano (Laranjeira et al., 2014). Alguns integrantes se manifestaram, dizendo que na cidade os números seriam maiores. O cenário motivou o esclarecimento de como se chegou a essa estatística. Nesse sentido, houve explanação da metodologia empregada no II Lenad e sua fidedignidade, assim como a explicação de possíveis variações por município, uma vez que foram verificadas pela Vigilância de Doenças Crônicas por Inquérito Telefônico (Brasil, 2011a).
Assim, o conteúdo da questão no 5 , que define a importância da epidemiologia do uso de drogas, foi contemplado neste momento. Para marcar a resposta correta, era necessário relacionar o fornecimento de subsídios para campanhas preventivas, com o conhecimento do consumo de drogas num determinada população e o conhecimento de novas tendências de uso de drogas como fatores que agregam importância aos estudos epidemiológicos. Entretanto, esta questão apresentou posterior redução na frequência de respostas corretas.

A literatura aponta que os estudos epidemiológicos possibilitam a afirmação de que o álcool é um dos maiores problemas de saúde pública do Brasil. Apesar de ser lícito, traz altos custos para a sociedade e, assim, a alta prevalência do uso excessivo no país requer atenção proporcional nos projetos de saúde. O consumo do álcool é composto por questões farmacológicas, individuais (físicas e psicológicas) e sociais (Senad, 2014; Victora et al., 2011).

O grupo também recebeu orientação sobre o conceito de dose-padrão no momento em que era explicado sobre o rigor das pesquisas epidemiológicas e, neste momento, ocorreu a pergunta de uma participante a respeito da dose-padrão, se essa seria a dose máxima que uma pessoa poderia ingerir antes de dirigir. Por isso, o conceito foi explicado e abordado o tema da Lei Seca de 2008 que seria posteriormente trabalhado no tópico Políticas Públicas. Foi dito na referida oportunidade, sobre como a mudança na lei contribuiu para uma recente redução do nível de alcoolemia permitido para dirigir. Neste sentido, é importante notar que a Lei Seca e outras medidas que objetivam reduzir o número de acidentes de trânsito no Brasil já mostraram seus efeitos com uma diminuição generalizada no comportamento de beber e dirigir da população (Oliveria et al., 2014).

Faz-se importante perceber que medidas de controle social são apontadas pelo Global Status Report on Alcohol and Health 2014 (WHO) como fatores determinantes do nível de vulnerabilidade ao uso nocivo do álcool da população. Aponta-se ainda que a eficácia deste tipo de política influencia positivamente na redução dos prejuízos causados pelo álcool (WHO, 2014).

Ainda sobre políticas públicas, há o tema da publicidade. Sobre tal assunto, diferentemente da regulação das propagandas referentes ao tabaco, a sociedade ainda não se posicionou sobre as pro- 
pagandas agressivas de cerveja, em sua maioria direcionadas aos jovens e vinculadas a esportes e eventos culturais (Pinsky, \& El Jundi, 2008). A legislação limita principalmente as bebidas com teor alcoólico maior do que $13 \%$ e tentativas de regulação por parte da Agência Nacional de Vigilância Sanitária (Anvisa) foram repetidamente derrubadas pela indústria e pela mídia (Victora et al., 2011). Este assunto foi tratado na questão $\mathrm{n}^{\circ} 6$, assinalando aumento do número de respostas certas no segundo momento.

Ao discutir as Políticas Públicas também ocorreram interferências positivas sendo que um Agente Comunitário de Saúde (ACS) relatou ter assistido recentemente na televisão que a venda de bebidas alcoólicas nos campeonatos estaduais de futebol foi liberada. $\mathrm{O}$ fato gerou um pequeno debate sobre como a sociedade deve lidar com a regulação e qual deve ser o papel da legislação. O tópico incentivou questionamentos sobre a questão cultural no uso de bebidas alcoólicas ao passo em que existe também a necessidade de o poder público impor limites e regras de consumo.

A questão $\mathrm{n}^{\circ} 4$ abordou exatamente a equivalência da dose-padrão em diferentes bebidas e apresentou a evolução mais relevante da pesquisa com a frequência de acertos tendo sido $42,5 \%$ superior no segundo momento da aplicação do questionário.

As bebidas alcoólicas podem conter diferentes concentrações de álcool e há uma quantidade específica de álcool puro denominada "dose-padrão", que equivale a cerca de 14 gramas ou 17,5 ml de álcool puro. Assim, existe a equivalência de bebidas alcoólicas que em quantidades diferentes correspondem a uma dose-padrão: $340 \mathrm{ml}$ de cerveja, $40 \mathrm{ml}$ de bebida destilada ou $140 \mathrm{ml}$ de vinho (Senad, 2014).

Ao realizar perguntas dirigidas aos participantes sobre a relação dos assuntos com o cotidiano, alguns profissionais afirmaram que os casos de uso problemático do álcool por vezes chegam à unidade de saúde como uma crise nervosa e, ao mesmo tempo, com grandes evidências de que o usuário está embriagado. Este exemplo fez referência ao conteúdo trabalhado na questão $\mathrm{n}^{\circ} 15$ que abordou a ambivalência no processo de mudança da pessoa que faz uso abusivo de bebida alcoólica, ou seja, pode ocorrer de ora aderir ao tratamento e ora evitá-lo. Outro ponto passível de debate com este comentário foram os diferentes modelos de assistência que podem ser oferecidos, como a proposta da Redução de Danos que teve sua definição abordada durante o curso e na questão $\mathrm{n}^{\circ} 14$ (Luppino et al., 2013; Oliveira, Andretta, Rigoni, \& Szupszynski, 2008).

Cabe ressaltar que a abordagem e o modelo de assistência oferecidos poderão acarretar riscos ao usuário, uma vez que, a não confirmação da embriaguez pelo próprio usuário, poderia demonstrar falta de crítica sobre o grave problema que o mesmo enfrenta e ocasionar a subnotificação dos casos de uso problemático de substâncias psicoativas (Almeida, Santana, Antunes, \& Silvino, 2014).

Outros apontamentos surgiram em meio às explanações e foram elucidados no decorrer do curso, todos foram anotados no diário de campo e alguns selecionados para este texto de modo a enriquecer a seção de discussão:

[...] o trabalho na APS é realizado, mas não existe sequência no tratamento (P4).

no CRAS, os alcoólatras não querem ficar com os loucos e os loucos não querem ficar com os alcoólatras [...] não tem como promover saúde com uma pessoa que não quer parar de beber (P16).

Tais trechos revelaram desconhecimento sobre a Rede de Atenção Psicossocial do município, tampouco a identificação do local de trabalho, pois o participante não se refere ao Centro de Referência de Assistência Social (CRAS) e sim ao CAPS, com funções bastante distintas. Em seguida, as expressões "loucos" e "alcoólatras" não deveriam ser utilizadas, pois são capazes de estigmatizar pessoas a partir dos problemas de saúde que enfrentam. Além disso, com tais denominações e a consideração da separação ou isolamento, a participante evidenciou a lógica manicomial que ainda se faz presente como proposta de tratamento em saúde mental e demonstrou desconhecimento com a atenção psicossocial proposta pelo SUS (Oliveira, 2009; Senad, 2014). Essas questões foram exaustivamente discutidas.

O comentário acerca do desejo do usuário parar ou não parar de beber evidencia despreparo na articulação entre o campo teórico e abordagem das pessoas portadoras de transtornos relacionados ao uso de álcool. Isso coincide com o que é tratado na questão $\mathrm{n}^{\circ} 11$ do questionário, em que se esperava o reconhecimento por parte do participante de que a ESF seria considerada a porta de entrada da APS. 
Em meio às participações destacou-se outro relato:

deveria ter uma lei em que a pessoa não tem o direito de aceitar ou não a internação; tem muita burocracia pra ser internado legalmente (P4).

O conteúdo deste relato reforçou novamente o despreparo na abordagem da pessoa com transtorno relacionado ao uso de álcool e outras drogas pelas equipes de saúde, visto que existe um descrédito com a APS em detrimento de uma lógica hospitalocêntrica. Sobre isso, o Global Status Report on Alcohol and Health 2014 (WHO) aponta dados que revelam a importância e efetividade da atuação dos profissionais da saúde que devem desenvolver e fornecer serviços de prevenção, tratamento e triagem. Vale ressaltar que o documento aponta a necessidade de capacitação das equipes proporcional à magnitude do problema enfrentado (WHO, 2014).

Espera-se, também, que a posição estratégica do profissional da APS na ESF possa contribuir para o enfrentamento das famílias frente ao uso problemático de bebida alcoólica e ao funcionamento da Rede de Atenção Psicossocial que deveria ter como objetivo, trabalhar de maneira geral a saúde do paciente, levando em consideração aspectos sociais, familiares e de trabalho (Brasil, 2016). Essas considerações foram apontadas nas questões $\mathrm{n}^{\circ} 16$ e $\mathrm{n}^{\circ} 17$ que apresentaram porcentagem final de $94,4 \%$ e $80,6 \%$ de respostas corretas, o que evidencia a assimilação deste conteúdo.

Em outro momento, um participante relatou sua percepção em relação às pessoas portadoras de transtornos relacionados ao uso de álcool e outras drogas:

existem casos que são conhecidos, como os que ficam na pracinha, mas tem também alguns que são abafados pela família (P1).

Considerando a família como elemento central da ESF, ela precisa ser entendida como protagonista do cuidado, corresponsabilizando-se pelo tratamento dos seus membros. Assim, tais percepções são importantes para adequar as formas de abordagens e as práticas profissionais daqueles que atuam na APS. Acredita-se que as equipes de SF (Saúde da Família) estão em uma posição estratégica em relação ao acolhimento de pessoas com uso problemático de bebida alcoólica porque a comunidade conhece e confia no seu trabalho (Brasil, 2012).
Os profissionais das equipes de SF têm condições de perceber tantos os casos de abuso de substância psicoativa que estão em destaque na comunidade quanto os casos em que há relativa negligência. Cabe ressaltar que essas características de cuidados primários à saúde da ESF também são apontadas como sendo uma possibilidade para que os povos atinjam uma vida produtiva e bons níveis de saúde (Brasil, 2002; 2012).

Os participantes comentaram:

[...] é só considerado alcoólatra aquele que está no fundo do poço; aquele que bebe três cervejas todo dia não é (P21).

o difícil não é reconhecer o alcoólatra, é fazer ele aceitar que é (P3).

[...] enquanto o quadro não se torna grave tanto em aspectos médicos ou psicossociais, o reconhecimento do uso problemático do álcool não se dá nem pelo usuário, nem pela família do mesmo (P16).

Segundo a Organização Mundial da Saúde (OMS), através do documento Self-help strategies for cutting down or stopping substance use: a guide (WHO, 2010b), não existe um nível seguro para o consumo de álcool especialmente se a pessoa bebe mais de duas doses por dia e se não deixa de beber pelo menos dois dias da semana (WHO, 2010b).

Vale ressaltar que o diagnóstico de um transtorno mental devido ao uso de álcool é um ato médico que será feito utilizando-se principalmente o CID-10 ou o DSM-V. Somado a isso, vale salientar que o principal modelo de tratamento adotado pelo SUS é baseado na estratégia de Redução de Danos, ou seja, o sucesso do tratamento não é atribuído apenas quando o usuário permanece em abstinência. A questão ${ }^{\circ} 14$ do questionário versou especificamente sobre este ponto e denotou que as práticas de redução de danos estão baseadas no esforço da preservação da vida, na tolerância e no não julgamento moral dos usuários. $\mathrm{O}$ alto índice de acertos $(84,2 \%)$ desta questão já no primeiro momento da aplicação dos questionários evidencia que esta lógica de tratamento já é de conhecimento de grande parte da equipe.

Dentre algumas práticas possíveis neste escopo estão: orientações sobre os riscos que o consumo de 
bebidas alcoólicas pressupõe; restrição do consumo exagerado e estabelecimento de situações sociais em que se faz uso de bebida alcoólica; sugestão para a definição da quantidade limite; o consumo de forma lenta, com intervalos longos e alternando bebidas alcoólicas com bebidas sem álcool; alimentação antes de ingerir bebidas alcoólicas; e não dirigir ou se colocar em situações de risco. Deste modo, a aceitação ou não aceitação do quadro de uso problemático do álcool não impedirá que o trabalho das equipes de SF se inicie e que seja eficiente (Luppino et al., 2013; Senad, 2014).

Os padrões de uso formam uma terminologia didática e com a possibilidade de descrever aspectos médicos e psicossociais com possíveis consequências decorrentes do uso do álcool. A OMS, ao publicar o Lexicon of Alcohol and Drug Terms, propôs nomenclaturas amplamente utilizadas no Brasil. O consumo moderado trata de um termo impreciso para um padrão de beber que implicitamente se contrapõe ao beber intenso. Significa beber quantidades moderadas e que não causam problemas. O beber social refere-se ao uso de bebidas alcoólicas de acordo com os costumes sociais e de maneira socialmente aceitável. Já o beber intenso excede as normas do beber social e é frequentemente definido por mais de três doses diárias ou acima de cinco doses por ocasião. $\mathrm{O}$ ato de beber que causa problemas, individuais ou coletivos, de saúde ou sociais é denominado beber problemático. O documento também define o bingedrinking como um padrão de ingestão intensa durante um período prolongado, escolhido para tal (WHO, 1994).

Entender os padrões de uso se faz importante principalmente para propostas de intervenção anteriores à instalação de um quadro de dependência do álcool, portanto, dados relevantes aos profissionais da atenção primária. O padrão de uso mais grave seria aquele que resulta no grande descontrole do bebedor além de alterações em sua vida particular e social por longo período denominado "alcoolismo", que se definido como uma alteração cerebral influenciada por fatores ambientais e que afeta o comportamento.

As ciências da saúde definem o alcoolismo como síndrome multifacetada. No Brasil, diretrizes para o tratamento da dependência do álcool preconizam a adoção do paradigma de redução de danos (RD) e o importante papel da APS. De modo sucinto, a RD é uma estratégia da saúde pública que busca reduzir os danos à saúde em consequência de práticas de risco. A implantação de estratégias deverá atentar para rela- ções intergrupais entre profissionais, usuários e seus familiares, pois evidencia-se que a abordagem dos profissionais de saúde ao dependente de álcool e drogas na APS não é satisfatória, e a política de RD, apesar de já ter sido adotada pelo Estado, parece não passar de uma proposta, pois ainda não faz parte da rotina nos serviços de APS (Reis, Silva, \& Andrade, 2010; Souza, Menandro, \& Menandro, 2015).

De acordo com o CID-10 e o DSM-V, o alcoolismo está estreitamente associado aos processos de tolerância e abstinência. As pessoas diagnosticadas com transtorno mental e comportamental relativo ao uso de álcool necessitam de quantidades progressivamente maiores para atingir o mesmo efeito e apresentam sintomas de abstinência alcoólica consumindo bebida alcoólica para aliviar ou evitar tais sintomas. Acerca destes assuntos, as questões $n^{\circ} 1$ e $n^{\circ} 3$ tiveram aumento do número de respostas corretas no segundo momento da aplicação.

Na dinâmica adotada de discussões teóricas e abertura para questionamentos e debates, ocorreu questionamento de um participante:

como tratar aquele paciente que já foi identificado, que a família já sabe do caso, mas que ele não vai fazer o tratamento? (P6).

Um importante apontamento sobre a participação da família no tratamento ocorreu na questão ${ }^{\circ} 19$ do questionário, em que a alternativa correta afirmava que a participação da família também seria positiva quando impões limites e regras mínimas e claras a serem cumpridas. Vale ressaltar que orientações educativas aos familiares podem ser importantes para esclarecer sobre o efeito bifásico do álcool (euforia e disforia) e sugerir momentos em que o diálogo com o familiar pode ser facilitado devido ao seu estágio motivacional. Estes temas foram abordados nas questões $\mathrm{n}^{\circ} 2$ e $\mathrm{n}^{\circ} 15$ do questionário e apresentaram respectivamente evoluções de $17,3 \%$ e $25,0 \%$ no número de respostas corretas considerando os dois momentos de aplicação do questionário.

Ainda sobre a questão $\mathrm{n}^{\circ} 19$ do questionário, que se refere à participação da família no tratamento, surgiu a indagação sobre promover mais estudos a respeito do tema e investigar a capacitação das equipes neste sentido visto que o item apresentou baixos percentuais de acertos ( $41,0 \%$ e $22,2 \%$ ) e revelou, ainda, uma redução do número de acertos após o curso. 
A relevância deste dado surgiu principalmente pelo papel estratégico das famílias nos cuidados de saúde e da corresponsabilização almejada entre equipe, usuário e familiares. Entretanto, outros estudos (Jorge et al, 2011; Nirettu-Pires, 2009) apontam que, apesar da visão complexa do usuário proposta pelo modelo de atenção da Saúde da Família, a formação dos profissionais ainda permanece fragmentada $\mathrm{e}$ restrita ao âmbito da disciplinaridade. De modo que a evidência da subjetividade e da singularidade de cada indivíduo, necessárias para o cuidado integral em saúde mental, necessita da transposição dos conceitos de patologia e diagnóstico para atendimentos com acolhimento e resolubilidade (Brasil, 2012).

Outro assunto discutido que também se refere às famílias trata-se das questões de hereditariedade. Possuir familiares com transtornos devido ao uso de álcool é um fator de risco. O Global Status Report on Alcohol and Health 2014 também abordou demandas relativas a gênero e alerta para que a saúde pública esteja atenta ao uso de álcool entre as mulheres, pois este número mostrou crescimento constante. $\mathrm{O}$ relatório sugeriu que o desenvolvimento econômico dos países influencia no consumo abusivo do álcool e que as pessoas com menor renda são mais vulneráveis a problemas relacionados ao consumo abusivo de álcool devido à falta de recursos para lidar com as consequências adversas. Em contraponto, quanto maior a riqueza econômica, maior o nível de consumo (WHO, 2014).

Quando o tema tratamento foi discutido, um participante com experiência em Pronto-Socorro relatou:

[...] a abordagem neste local costumava se resumir à aplicação de soro glicosado ou apenas soro quando o paciente é diabético... ainda que, por vezes, as pessoas que chegam embriagadas com grande frequência a este serviço muitas vezes não são medicadas. Às vezes, está com uma ferida, um corte, e o corte nem fechou e eles já estão lá de novo com outro... alguns casos que a pessoa não tem acompanhante o motorista da ambulância já leva embora direto (P22).

Estes dados foram direcionados para reflexões no âmbito da Rede de Atenção Psicossocial com objetivo de entendimento amplo, crítico e de questionamentos sobre os papéis das diferentes instituições e dinâmicas de encaminhamentos de referência e contrar- referência no município. Os fluxos e funcionamentos da rede têm importância crucial na resolubilidade dos casos e na qualidade dos serviços prestados, visto que os profissionais de saúde devem atuar avaliando não somente as demandas de sua unidade, mas com atenção à qualidade dos encaminhamentos, orientações e às especificidades de cada setor (Campos, \& Domitti, 2007; Moretti-Pires, 2009; Senad, 2014).

Em outro relato:

[...] acho errado que as pessoas com problemas com bebida alcoólica fiquem internadas juntas com as pessoas com problemas mentais (P23).

Mediante os relatos, observa-se a real necessidade de encontros que versem sobre o movimento da luta antimanicomial e sobre outras possibilidades de tratamento, incluindo dados sobre a Lei $n^{\circ} 10.216$, de 6 de abril de 2001 e sobre a necessidade de criação de leitos psiquiátricos em hospitais gerais (Brasil, 2011b; Pitta, 2011).

Como parte programada do curso, o este Audit (Babor, Higgins-Biddle, Saunders, \& Monteiro, 2001; Saunders, Aasland, Babor, De La Fuente, \& Grant, 1993; Senad, 2014) foi apresentado aos cursistas. Ocorreu a apresentação dos dados técnicos do teste e orientações sobre a correta aplicação e fundamentação teórica do mesmo. Os participantes foram encorajados a responder ao teste ou aplicá-lo em outra pessoa. Alguns relataram autoaplicação e outros afirmaram que aplicaram o questionário com familiares. Alguns cursistas não aceitaram compartilhar os resultados. Assim, o grupo foi incentivado a falar sobre o que achou da possibilidade de utilização deste teste no trabalho cotidiano.

O primeiro comentário foi sobre a fidedignidade quando um participante perguntou sobre como fazer o teste se o paciente não fala as informações verdadeiras. Outro participante questionou se o teste detectaria o grau de dependência. $O$ grupo foi orientado a recorrer ao material didático para verificar a correção, as pontuações e as possibilidades de intervenção de acordo com a zona de risco.

Seria importante salientar que alguns profissionais relataram que o teste poderia ser de grande utilidade no cotidiano do trabalho e enfatizaram sua facilidade de aplicação e o pouco tempo gasto para tal. Coube ressaltar também que os problemas relacionados ao consumo de álcool ultrapassam as questões de 
saúde e que talvez fosse interessante a utilização do teste mesmo com usuários com os quais a equipe não suspeite do uso nocivo.

Sobre as dificuldades no trabalho, antes da realização do curso, na questão $\mathrm{n}^{\circ} 18$ do questionário, $73,7 \%$ dos participantes afirmaram que a somatória da falta de tempo ou da falta de motivação com mudanças frequentes das equipes e com o excesso de outras atividades obrigatórias são fatores que interferem na capacidade de acolhimento às pessoas com uso problemático do álcool.

Com relação ao tratamento, mesmo após o acolhimento, o acompanhamento dessas pessoas nem sempre é assegurado, segundo o relato:

[...] não é fácil realizar encaminhamentos, porque não tem muitos grupos,... de convivência. A gente encaminha para o CAPS (P10).

As maiores dificuldades na direção da APS estão relacionadas à fragmentação da atenção à saúde. Recentemente, alguns estudos têm explorado as características da lógica matricial como maneira não verticalizada de organização do sistema, uma possibilidade de criação de espaços de comunicação e criação de estratégias para lidar com os problemas de saúde (Campos, \& Domitti, 2007; Cunha, \& Campos, 2011). Denota-se no relato destacado a pertinência da lógica matricial para a atenção à saúde no caso das pessoas portadoras de transtornos relacionados ao uso de álcool e outras drogas. As experiências de encaminhamento, mesmo que parciais, foram compreendidas como exitosas na utilização do sistema de referência e contrarreferência com o CAPS do município.

Espera-se que os encaminhamentos de referência e contrarreferência sejam mais adequados, principalmente com relação à proporcionalidade da gravidade da situação dos usuários, sendo coerente com o nível de atenção dos atendimentos previstos. Também há expectativa do fortalecimento e estruturação da Rede de Atenção Psicossocial, bem como das características de abordagens com referência na Redução de Danos.

Segundo o Ministério da Saúde (MS), a Política para Atenção Integral a Usuários de Álcool e Outras Drogas (Brasil, 2003) ressalta que o diagnóstico e tratamento precoces da dependência do álcool são fatores fundamentais no prognóstico deste transtorno, de modo a conclamar perspectivas globais de prevenção e promoção de saúde. Portanto, trata-se de um problema de saúde pública, que necessita de fundamentos práticos e teóricos para intervenções baseadas em evidências científicas, mas que sejam aplicáveis frente às realidades encontradas nos serviços de APS.

\section{Considerações finais}

A avaliação da efetividade do curso por meio de questionários alcançou parâmetros para mensurar seu impacto positivo nas apreensões de conteúdo relativos à abordagem e ao conhecimento frente à problemática do consumo de bebidas alcoólicas, visto que a educação continuada em saúde tem evidente potencial de política pública, e como tal deve apresentar justificativas para sua regulamentação. Vale ressaltar que o envolvimento e participação dos profissionais também garantiram a viabilidade deste modelo de intervenção.

A opção em proporcionar um espaço para reflexões críticas do cotidiano teve como desafio a articulação dos conteúdos científicos com as rotinas de trabalho na APS, o que exigiu uma característica multiprofissional e interdisciplinar em seu planejamento. A proposta apresenta, ainda, uma possibilidade de atuação do profissional da Psicologia na rede municipal de saúde com foco nos constructos da Promoção da Saúde.

O curso possibilitou a reunião dos profissionais participantes em um só local, viabilizando o diálogo e a expressão de satisfação, queixas e até mesmo reflexões críticas sobre o trabalho desenvolvido. O modelo de intervenção também evidenciou potencialidades para o diálogo de temas e situações que transcenderam ao esperado. O grupo apontou deficiências quanto ao conhecimento do processo histórico da Psiquiatria no Brasil e da Saúde Mental até os dias atuais, visto a presença de relatos com viés de estigmatização aos portadores de transtornos mentais.

De modo geral, os relatos dos profissionais participantes do curso acusam o funcionamento adequado da rede de saúde municipal no que tange à gestão e especificidade das unidades, entretanto, as dificuldades são em grande parte apontadas para a maneira de trabalhar a individualidade e peculiaridade dos casos. O que aponta a necessidade frequente de projetos de educação continuada, segundo as demandas dos profissionais, bem como do desenvolvimento de pesquisas posteriores que identifiquem fatores que possam influenciar a dinâmica do uso problemático do álcool na esfera municipal. 


\section{Referências}

Agência Nacional de Saúde Suplementar - ANS (2011). Manual técnico para promoção da saúde e prevenção de riscos e doenças na saúde suplementar (4a ed.). Brasília, DF: o autor.

Almeida, E. G., Santana, C. J., Antunes, F., \& Silvino, M. C. S. (2014). Busca ativa e toxicovigilância: diminuindo subnotificação de casos de drogas de abuso. In $2^{\circ}$ Congresso Paranaense de Saúde Pública (p. 286). Londrina, PR: Instituto de Estudos em Saúde Coletiva.

Babor, T. F., Higgins-Biddle, J. C., Saunders, J. B., \& Monteiro, M. G. (2001). The alcohol use disorders identification test: guidelines for use in primary care (2nd ed.). Geneva: World Health Organization.

Bau, C. H. D. (2002). Estado atual e perspectivas da genética e epidemiologia do alcoolismo. Ciência e Saúde Coletiva, 7(1), 183-190. https://doi.org/10.1590/S1413-81232002000100017

Brasil. (2003). A política do Ministério da Saúde para atenção integral a usuários de álcool e outras drogas. Brasília, DF: Ministério da Saúde.

Brasil. (2011b, 9 abr.). Lei No 10.216, de 6 de abril de 2001. Dispõe sobre a proteção e os direitos das pessoas portadoras de transtornos mentais e redireciona o modelo assistencial em saúde mental. Diário Oficial da União.

Brasil. (2006). Ministério da Saúde, Secretaria de Atenção à Saúde. Política nacional de atenção básica. Brasília, DF: o autor.

Brasil. (2002). Ministério da Saúde. Secretaria de Políticas de Saúde. As cartas da promoção da saúde (Série B. Textos básicos em saúde). Brasília, DF: Ministério da Saúde.

Brasil. (2011a). Ministério da Saúde. Vigitel Brasil 2011: vigilância de fatores de risco e proteção para doenças crônicas por inquérito telefônico: estimativas sobre frequência e distribuição sociodemográfica de fatores de risco e proteção para doenças crônicas nas capitais dos 26 estados brasileiros e no Distrito Federal em 2011. Brasília, DF: o autor.

Brasil (2012). Portal da Saúde SUS. Departamento de Atenção Básica. Como funciona? Equipe de saúde da família. Brasília, DF: o autor. Recuperado de http://dab.saude.gov.br/portaldab/smp_como_funciona.php?conteudo=esf

Brasil (2002, 20 fev.). Portaria $N^{\circ} 336$, de 19 de fevereiro de 2002. Define e estabelece diretrizes para o funcionamento dos Centros de Atenção Psicossocial. Diário Oficial da União.

Campos, G. W. S., \& Domitti, A. C. (2007). Apoio matricial e equipe de referência: uma metodologia para gestão do trabalho interdisciplinar em saúde. Cadernos de Saúde Pública, 23(2), 399-407. https://doi.org/10.1590/S0102-311X2007000200016

Cunha, G. T., \& Campos, G. W. S. (2011). Apoio matricial e atenção primária em saúde. Saúde e Sociedade, 20(4), 961-970. httpa://doi.org/10.1590/S0104-12902011000400013

Falkembach, E. M. F. (1987). Diário de campo: um instrumento de reflexão. Contexto e Educação (Ijuí), 7(2), 19-24.

Jorge, M. S. B., Pinto, D. M., Quinderé, P. H. D., Pinto, A. G. A., Sousa, F. S. P., \& Cavalcante, C. M. (2011). Promoção da saúde mental: tecnologias do cuidado: vínculo, acolhimento, co-responsabilização e autonomia. Ciência \& Saúde Coletiva, 16(7), 3051-3060. https://doi.org/10.1590/S1413-81232011000800005

Laranjeira, R., Madruga, C., \& Pinsky, I. (2014). II Levantamento Nacional de Álcool e Drogas (LENAD). São Paulo: Instituto Nacional de Ciência e Tecnologia para Políticas Públicas de Álcool e Outras Drogas.

Lima, A. I. O. (2014). Suporte ao uso de álcool e drogas na atenção primária: um estudo com equipes de Natal/RN (Dissertação de mestrado). Universidade Federal do Rio Grande do Norte, Natal, RN.

Lima, T. C. S., Mioto, R. C. T., \& Dal Prá, K. R. (2007). A documentação no cotidiano da intervenção dos assistentes sociais: algumas considerações acerca do diário de campo. Textos \& Contextos (Porto Alegre), 6(1), 93-104. Recuperado de http://revistaseletronicas.pucrs.br/ojs/index.php/fass/article/view/1048/3234

Luppino, J., Nunes, M. C., Silva, M. C., Piquini, M. K., Fornari, P. F., Franco, R. L. et al. (2013). Abordagem de usuários de drogas e redução de danos: atualização do conhecimento de agentes comunitários de saúde em Sorocaba-SP. Revista da Faculdade de Ciências Médicas de Sorocaba, 15(4), 116-120. Recuperado de https://revistas.pucsp.br/ index.php/RFCMS/article/view/15303/pdf

Moretti-Pires, R. O. (2009). Complexidade em Saúde da Família e formação do futuro profissional de saúde. Interface (Botucatu), 13(30), 153-166. https://doi.org/10.1590/S1414-32832009000300013 
Oguisso T. (2000). A educação continuada como fator de mudanças: visão mundial. Nursing, 3(20), 22-9.

Oliveira, A. S., Pedro, J. B., Mamlak, L., Oliveira, L. S., Mendonça, L. O., Santiago, M. F. et al. (2014). A nova lei seca: mudanças ocasionadas pela antiga e nova lei no código de trânsito brasileiro. Caderno de Graduação, 2(2), 137-146. Recuperado de: https://periodicos.set.edu.br/index.php/cadernohumanas/article/view/1683/959

Oliveira, M. S., Andretta, I.,Rigoni, M. S., \& Szupszynski, K. P. R. (2008). A entrevista motivacional com alcoolistas: um estudo longitudinal. Psicologia: Reflexão e Crítica, 21(2), 261-266. https://doi.org/10.1590/S0102-79722008000200011

Oliveira, W. F. (2009). Éticas em conflito: reforma psiquiátrica e lógica manicomial. Cadernos Brasileiros de Saúde Mental, 1(2), 42-56. Recuperado de http://incubadora.periodicos.ufsc.br/index.php/cbsm/article/view/1126/1362

Olschowsky, A., Wetzel, C., Fernando, J. S., Pinho, L. B., \& Camatta, M. W. (2014). Avaliação das parcerias intersetoriais em saúde mental na estratégia saúde da família. Texto \& Contexto - Enfermagem, 23(3), 591-599. https://doi.org/10.1590/0104-07072014001970012

Organização Panamericana de La Salud - OPS. (1979). Educación contínua: guia para la organización de programas de educación continua para personal de salud. Washington, DC: o autor.

Pereira, I. B., \& Lima, J. C. F. (2006). Dicionário da educação profissional em saúde. Rio de Janeiro: Fundação Oswaldo Cruz, Escola Politécnica de Saúde Joaquim Venâncio.

Pinsky, I., \& El Jundi, S. A. (2008). O impacto da publicidade de bebidas alcoólicas sobre o consumo entre jovens: revisão da literatura internacional. Revista Brasileira de Psiquiatria, 30(4), 362-74. https:// doi.org/10.1590/S1516-44462008005000015

Pitta, A. M. F. (2011). Um balanço da reforma psiquiátrica brasileira. Ciência \& Saúde Coletiva, 16(12), 4593-4596. https://doi.org/10.1590/S1413-81232011001300002

Reis, I. F., Silva, J. L. L., \& Andrade, M. (2010). Utilização da política de redução de danos de álcool e outras drogas em saúde da família. Informe-se, 6(2), 16-19. Recuperado de http:/ /www.uff.br/promocaodasaude/drogas.2010.pdf

Saunders, J. B., Aasland, O. G., Babor, T. F., De la Fuente, J. R., \& Grant, M. (1993). Development of the alcohol use disorders identification test (AUDIT):WHO collaborative project on early detection of persons with harmful alcohol consumption-II. Addiction, 88(6), 791-791. Recuperado de https://doi.org/10.1111/j.1360-0443.1993.tb02093.x

Secretaria Nacional de Políticas sobre Drogas - Senad. (2014). SUPERA: Sistema para detecção do Uso abusivo e dependência de substâncias Psicoativas: Encaminhamento, Intervenção Breve, Reinserção social e Acompanhamento. Brasília, DF: o autor. Recuperado de http://www.supera.senad.gov.br

Silva, C. J. (2005). Impacto de um curso em diagnóstico e tratamento do uso nocivo e dependência do álcool sobre a atitude e conhecimento de profissionais da rede de atenção primária à saúde (Tese de doutorado). Escola Paulista de Medicina, Universidade Federal de São Paulo, São Paulo, SP.

Silva, G. M., \& Seiffert, O. M. L. B. (2009). Educação continuada em enfermagem: uma proposta metodológica. Revista Brasileira de Enfermagem, 62(3), 362-366. http://dx.doi.org/10.1590/S0034-71672009000300005

Souza, K. L. (2014). Avaliação do conhecimento de profissionais da atenção básica sobre crack, álcool e outras drogas (Monografia). Universidade de Brasília, Brasília, DF.

Souza, L.G.S.; Menandro, M.C.S. \& Menandro, P.R.M. (2015). O alcoolismo, suas causas e tratamento nas representações sociais de profissionais de Saúde da Família. Physis: Revista de Saúde Coletiva, 25(4), 1335-1360

Spinillo, A. G. (1994). Estudos de treinamento e variações experimentais. Temas em Psicologia, 2(3), 43-57. Recuperado de http://pepsic.bvsalud.org/scielo.php?script=sci_arttext\&pid=S1413-389X1994000300006

Victora,C.G.,Barreto,M.L.,Leal,M.,Monteiro,C.A.,Schmidt,M.I.,Paim,J.etal.(2011).Healthconditionsandhealth-policy innovations in Brazil: the way forward. Lancet, 377(9782), 90-102. https://doi.org/10.1016/S0140-6736(11)60055-X

World Health Organization - WHO. (2014). Global status report on alcohol and health 2014. Geneva: the author.

World Health Organization - WHO. (2010a). Global strategy to reduce the harmful use of alcohol. Geneva: the author.

World Health Organization - WHO. (1994). Lexicon of alcohol and drug terms. Geneva: the author.

World Health Organization - WHO. (2010b). Self-help strategies for cutting down or stopping substance use: a guide. Geneva: the author. 


\section{Fernando Gomes Reis}

Psicólogo pela Universidade Estadual de Maringá (UEM) e Mestrando em Promoção de Saúde pela Universidade de Franca, Franca, SP. Brasil.

E-mail: gomesreisfernando@gmail.com

Everson Cardozo Machado

Estatístico pela Universidade Federal Fluminense (UFF), Niterói, RJ. Brasil.

E-mail: eversoncmachado@hotmail.com

Mônica de Andrade

Doutora em Ciências Biológicas pela Universidade Federal de São Carlos (UFSCAR), Bióloga, docente do Programa de Pós-Graduação Stricto Sensu Promoção de Saúde da Universidade de Franca, Franca, SP, Brasil.

E-mail: mmonicandrade@gmail.com

\section{Glória Lúcia Alves Figueiredo}

Doutora pela Universidade de São Paulo (USP), Enfermeira, docente do Programa de Pós-Graduação Stricto Sensu Promoção de Saúde da Universidade de Franca, Franca, SP, Brasil.

E-mail: gloria.figueiredo@unifran.edu.br

Endereço para envio de correspondência:

Fernando Gomes Reis.

Rua Presidente Antônio Carlos, 237, Sala 06. CEP 37900-092.

Passos - MG, Brasil.

Recebido 01/03/2016

Reformulação 13/03/2017

Aprovado 05/04/2017

Received 03/01/2016

Reformulated $03 / 13 / 2017$

Approved 04/05/2017

Recebido 01/03/2016

Reformulado 13/03/2017

Aceptado 05/04/2017

Como citar: Reis, F. G., Machado, E. C., Andrade, M., \& Figueiredo, G. L. A. (2017). Conhecimento e abordagens acerca do uso problemático do álcool. Psicologia: Ciência e Profissão, 37(2), 335-348. https://doi.org/10.1590/1982-370300852016

How to cite: Reis, F. G., Machado, E. C., Andrade, M., \& Figueiredo, G. L. A. (2017). Knowledge and approaches on the issue of alcohol consumption. Psicologia: Ciência e Profissão, 37(2), 335-348. https://doi.org/10.1590/1982-370300852016

Cómo citar: Reis, F. G., Machado, E. C., Andrade, M., \& Figueiredo, G. L. A. (2017). El Conocimiento y las propuestas presentadas acerca del consumo problemático de alcohol. Psicologia: Ciência e Profissão, 37(2), 335-348. https://doi.org/10.1590/1982-370300852016 